\title{
Alterations of color vision and central visual field in patients with Vogt-Koyanagi-Harada syndrome
}

\author{
Peizeng Yang • Min Sun $\cdot$ Xiaoli Liu $\cdot$ Hongyan Zhou • \\ Wang Fang $\cdot$ Li Wang $\cdot$ Aize Kijlstra
}

Received: 11 April 2011 / Accepted: 28 November 2011 /Published online: 2 February 2012

(C) The Author(s) 2012. This article is published with open access at Springerlink.com

\begin{abstract}
Purpose To investigate the changes of color vision and central visual field in a cohort of patients with Vogt-KoyanagiHarada (VKH) syndrome.

Methods Sixteen VKH patients (32 eyes) were enrolled in this study. All the patients were treated with immunosuppressive agents. The best visual acuity, visual field testing and color vision testing were available from the records in all these patients at different time points, i.e. before treatment and 1 month ( \pm 7 days), 3 months ( \pm 15 days), 6 months ( \pm 20 days) and 12 months ( \pm 30 days) after treatment.

Results All patients showed active intraocular inflammation at their first visit. A decreased visual acuity, abnormality of color vision and abnormal visual field were observed at presentation. Visual acuity and color vision rapidly improved at 1 and 3 months and gradually improved thereafter. Visual field defects significantly improved at 6 months and gradually improved thereafter. However, visual field defects were still observed in $27.5 \%$ of the tested patients following a 12-month treatment. Color vision returned to the normal level only in about one-third of these patients at this time point.
\end{abstract}

P. Yang $(\bowtie) \cdot$ M. Sun $\cdot X$. Liu

The First Affiliated Hospital of Chongqing Medical University, Chongqing Key Laboratory of Ophthalmology,

Youyi Road 1,

Chongqing 400016, People's Republic of China

e-mail: peizengycmu@126.com

P. Yang $\cdot$ M. Sun $\cdot$ H. Zhou $\cdot$ W. Fang $\cdot$ L. Wang

Zhongshan Ophthalmic Center, Sun Yat-sen University,

Guangzhou, People's Republic of China

A. Kijlstra

Eye Research Institute Maastricht, Department of Ophthalmology,

University Hospital Maastricht,

Maastricht, the Netherlands
Conclusions Visual function was severely impaired in VKH patients with active uveitis but rapidly improved following immunosuppressive therapy. Visual fields are much more severely affected by the disease than visual acuity and its improvement lagged behind that of visual acuity and color vision.

Keywords Vogt-Koyanagi-Harada syndrome · Color vision · Visual field

\section{Introduction}

Vogt-Koyanagi-Harada (VKH) syndrome has generally been considered as an autoimmune disease against melanocytes. It is characterized by choroiditis at the onset of disease and a granulomatous uveitis at recurrence. A variety of extraocular manifestations are associated with this syndrome [1-4]. It is more prevalent in certain pigmented races, such as Asians and Native Americans [1-4]. Visual prognosis is generally favorable when prompt diagnosis was made and appropriate immunosuppressive treatment was installed $[2,5,6]$.

The central visual acuity is considered to be an important measurement of visual function and is an important indicator of visual outcome in patients with VKH syndrome [2, 5]. However, in our clinical experience, some patients with good or even normal central visual acuity still complain of visual disturbances such as visual field defects or abnormal color vision. In a recent study, we have revealed that certain VKH patients with normal visual acuity have overt abnormalities when tested by multifocal electroretinography [7]. Therefore, visual acuity measurement alone is not a sufficient parameter to accurately evaluate the retinal, especially the macular function. Central visual field and color vision 
have been shown to be important parameters for visual function [8]. In this study, we evaluated the alterations of color vision and central visual field in patients with VKH syndrome to better understand the effects of disease and treatment on visual function.

\section{Material and methods}

This retrospective study included patients diagnosed as VKH syndrome between January 1, 2006 and March 20, 2008. The diagnosis of VKH was based on detailed history and clinical examinations described in an earlier study [5]. All patients included in this study met the revised criteria for VKH syndrome by the International Nomenclature Committee [9]. A history or the presence of exudative retinal detachment and pigmentary changes involving the macular area was inquired or observed in all of the patients at first visit or follow-up visit in our hospital. Hereditary color vision defects were carefully inquired in each patient and no color vision defects were reported by any of them. Patients with conditions which might alter the color vision and visual field values, such as a history of glaucoma, presence of increased intraocular pressure, overt media opacity, posterior synechiae, a history of previous ocular surgery, or diabetes were excluded from the study.

The patients were followed up and examined using Snellen charts at each visit following treatment. Visual field testing and color vision testing were available from the records in all these patients at different time points, i.e. before treatment and 1 month ( \pm 7 days), 3 months ( \pm 15 days), 6 months ( \pm 20 days) and 12 months ( \pm 30 days) after treatment. These tests were performed in the routine facilities of the institute and the technicians administering the test were unaware of the diagnosis of the patients. A thorough ophthalmological examination, including slit-lamp biomicroscopy and ophthalmoscopy through a dilated pupil, was performed in all patients. For the purpose of statistical analysis, Snellen chart visual acuity was converted to the logarithm of the minimal angle of resolution (log MAR) value [10]. Visual fields were tested with the Humphrey Field Analyzer Program 30-2 according to the Humphrey perimetry manual. The efficiency of fixation of the subjects was constantly monitored by an experienced perimetrist. The mean deviation (MD, in decibels) and corresponding $P$ value of each field analysis was noted. The FarnsworthMunsell (FM) 100-hue test under standard illuminant C conditions was performed to detect color vision defects [8].

For purpose of statistical analysis, normal visual acuity was defined as best-corrected visual acuity (BCVA) with equal to or better than 0.8 in this study. Visual fields with false-positive responses of more than $15 \%$, false-negative responses, or a rate of fixation loss of more than $20 \%$ were not included in this analysis. In this study, we used MD and $P$ value to evaluate the visual field abnormality. MD value reflected the extensive damage of the retina. The more severe the retina damage was, the lower value of MD. Each $P$ value corresponds to a MD value. The $P$ value indicates the probability of that MD being found in the normal population. Color vision scores were determined using the FM 100 testing which was performed in a dark room with test materials viewed under standard illuminant conditions. Subjects used near vision correction, if needed, and were given 2 min to complete each of the four test boxes. The approximate distance from the subjects' eyes to the test materials was 30 to $50 \mathrm{~cm}$. Total error score was calculated according to the manufacturer's recommendations, and the square root of the total error score was used in analyses. A high value reflects a defect of color vision. For normal color vision values, we chose data from a population of healthy Chinese individuals previously reported by Huang and associates [11] who also measured the color vision with the FM 100 test.

The study followed the tenets of the World Medical Association Declaration of Helsinki. Informed consent was obtained from all patients prior to these aforementioned examinations and treatment with immunosuppressive agents.

The data were collected from the patients and were subsequently entered into a computer-based data bank. Statistical analysis was performed using SAS for windows XP. The ANOVA was used to test the significance of the mean values before treatment and the values at 1, 3, 6 and 12 months after treatment. A $P$ value less than or equal to 0.05 was considered statistically significant.

\section{Results}

This retrospective study included 32 eyes of 16 patients. There were nine males and seven females in the study patients. The mean age was $33.2 \pm 9.6$ years, ranged from 21 to 50 years old. All the patients with VKH syndrome had an active intraocular inflammation at their first visit and were treated with corticosteroids in combination with other immunosuppressive agents such as cyclosporine or chlorambucil. The ocular findings included diffuse choroiditis, exudative retinal detachment, optic disk edema or overt granulomatous anterior uveitis in association with 'sun-set glow' fundus and nummular chorioretinal depigmented scars.

The BCVA ranged from $20 / 250$ to $20 / 25$ (median $B C V A=20 / 71$ ) on Snellen charts, and the mean $\log$ MAR value was $0.72(\mathrm{SD}=0.42)$ before treatment. One month after treatment, the BCVA ranged from 20/200 to 20/16 (median $\mathrm{BCVA}=20 / 33$ ), and the mean $\operatorname{logMAR}$ value was 
$0.38(\mathrm{SD}=0.26)$, significantly lower than that before treatment $(P=0.026)$. Three months after treatment, the BCVA ranged from $20 / 80$ to $20 / 15$ (median $B C V A=20 / 28$ ), and the mean $\log$ MAR value was $0.18(\mathrm{SD}=0.10 ; P=0.012)$. The $\mathrm{BCVA}$ continued to improve at 6 months (median $\mathrm{BCVA}=$ $20 / 24$, mean $\log \mathrm{MAR}=0.12 \pm 0.06, P<0.001)$ and 12 months (median $\mathrm{BCVA}=20 / 21$, mean $\log \mathrm{MAR}=0.08 \pm 0.07, P<0.001$; Fig. 1). At the final follow-up, $81.3 \%$ of the eyes had a BCVA of $\geq 20 / 25$ and only $6.3 \%$ of the eyes had a BCVA below $20 / 40$.

FM 100-hue testing showed that the mean total error score (TES) in these patients was $365(\mathrm{SD}=135)$ before treatment. One month after treatment, the mean TES decreased to 178 $(\mathrm{SD}=91)$, showing a significant improvement $(P=0.029)$. The mean TES was further improved at 3 months $(124 \pm 56$, $P<0.001), 6$ months $(78 \pm 46, P<0.001)$ and 12 months (52 \pm $36, P<0.001$; Fig. 2). However, the mean TES was still abnormal in $65.6 \%$ of the tested patients at 12 months following treatment.

The MD measured with the Humphrey Field Analyzer ranged from 6.82 to $26.02 \mathrm{db}$, with a mean value of $15.62 \mathrm{db}$ $(\mathrm{SD}=6.98)$ in the VKH patients before treatment. One month after treatment, the MD value ranged from 6.20 to $17.59 \mathrm{db}$, with a mean value of $13.64(\mathrm{SD}=5.38 ; P=0.102)$. Three months later, the mean $\mathrm{MD}$ value was $8.20 \mathrm{db}(\mathrm{SD}=2.80$; $P=0.087)$. The mean MD value was significantly improved at 6 months $(4.34 \pm 1.58, P=0.026)$ and 12 months $(3.37 \pm 1.21$, $P=0.013$; Fig 3). At 12 months following treatment, the MD value returned to normal levels in $72.5 \%$ of these patients. The mean $P$ value was lower than $1.52 \%$ in the VKH patients before treatment. One and 3 months after treatment, the mean $P$ value was lower than $0.72 \%(P=0.448)$ and $0.76 \%$ $(P=0.426)$, respectively. At 6 months after treatment, the mean $P$ value was lower than $2.32 \%$, which was slightly higher than that before treatment although there was no statistical difference $(P=0.325)$. After 12-month treatment, the mean $P$ value was lower than $3.86 \%$, which was significantly higher than that before treatment $(P=0.032)$.

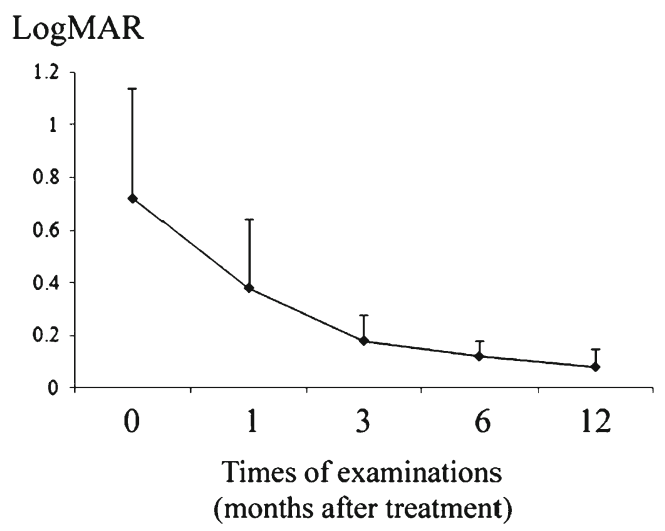

Fig. 1 Time course of best-corrected visual acuity (in terms of Log$M A R$ ) following immunotherapy in VKH eyes, the plots mean the average of the test, the error bars mean standard deviation: SD

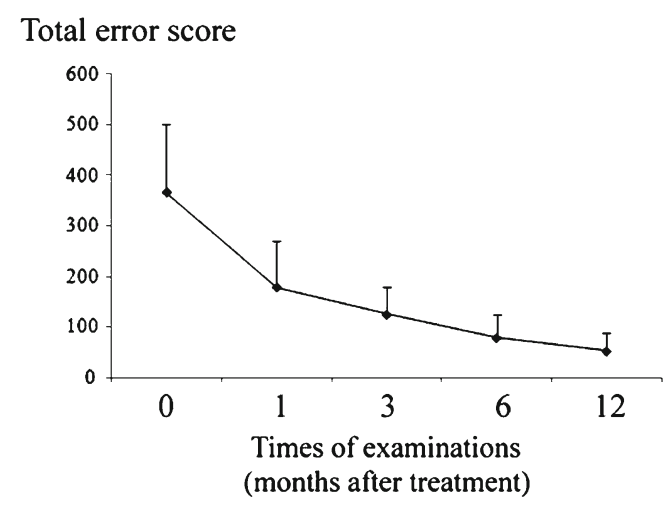

Fig. 2 Time course of color vision changes (in terms of total error score, TES) following immunotherapy in VKH eyes, the plots mean the average of the test, the error bars mean standard deviation: SD

\section{Discussion}

In this study, we evaluated the visual function of VKH patients with active uveitis before and after treatment with immunosuppressive agents using visual acuity, color vision and visual field. Our results showed that the patients displayed striking abnormalities in these three parameters before treatment, reflecting a severely impaired visual function in active VKH syndrome. An improvement of visual acuity and color vision was rapidly achieved at 1 and 3 months, followed by a gradual recovery at 6 and 12 months after treatment. For the visual field results, a marked amelioration was observed at 6 months and thereafter following treatment. These results show that there is a substantial recovery of visual function in VKH patients following appropriate immunosuppressive treatment. Among the three tested parameters, recovery of visual acuity and color vision preceded an improvement of visual field.

Various studies have shown that visual acuity at presentation is an important prognostic indicator of final visual acuity in patients with VKH syndrome $[2,5]$. Approximately $60-80 \%$ of patients could achieve a visual acuity of $20 / 40$ or better $[2,5,6]$. In our patients, we found that more than $90 \%$ had a visual acuity of $20 / 40$ or better at the final

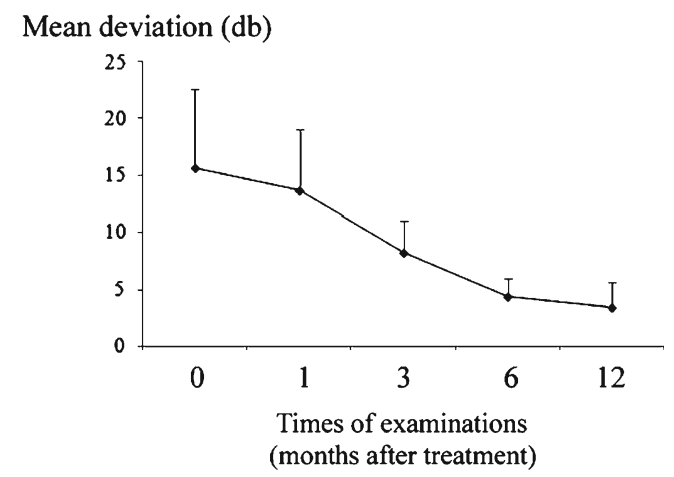

Fig. 3 Time course of visual field changes (in terms of mean deviation, MD) following immunotherapy in VKH eyes, the plots mean the average of the test, the error bars mean standard deviation: SD 
follow-up. Furthermore, a rapid decrease of the $\log$ MAR value was observed at 1 and 3 months following treatment, indicating a prompt recovery of visual acuity in $\mathrm{VKH}$ patients following appropriate treatment. This result is generally consistent with that reported by Rubsamen et al. [12].

A number of tests have been designed for color vision screening and grading. The Farnsworth-Munsell 100-hue test is designed to measure hue discrimination ability at a constant value and chroma and is considered an excellent choice in evaluating color vision defects [13].To rule out other causes of color vision defects, we excluded patients with conditions which could influence the color vision. Interestingly, we found that abnormal color vision was present in all the examined VKH patients with active uveitis. As color vision generally reflects the function of retinal cone cells [14], it is reasonable to presume that these cells were severely involved during VKH syndrome. Our results are in agreement with previous reports in which abnormal color vision is common in retinochoroidal or optic nerve disorders, such as diabetic retinopathy, tilted disc syndrome, optic neuritis and primary open angle glaucoma [15-18]. Abnormal color vision has also been reported in birdshot choroiretinopathy [19]. It is unknown whether abnormal color vision is a universal finding of uveitis involving the choroid and retina. Further study is required to clarify this issue. Similar to visual acuity, color vision rapidly improved after immunosuppressive treatment.

Visual field is another important parameter to evaluate visual function. Our study showed severe visual field abnormalities in VKH patients before treatment as evidenced by severely decreased MD value and $P$ value. Visual field has been shown to reflect the function of the retina, choroid and optic nerve [20]. There are numerous field abnormalities observed in ocular fundus diseases. The impaired visual field is generally in accordance with the inflammatory damage to the choroid and its adjacent tissues observed in this syndrome. Clinically, this disease is characterized by choroiditis, chorioretinitis and neuroretinitis at its first attack [2, 5]. Pathologically, marked losses of melanocytes and infiltration of lymphocytes in the choroid, and destruction or hyperplasia of retinal pigment epithelium (RPE) have been described in VKH syndrome [21]. In addition, fundus fluorescein angiography [22], indocyanine green angiography [23] and optical coherence tomography [24] also showed a variety of changes in the choroid, retina and RPE. Similar to visual acuity and color vision, central visual field also significantly improved following appropriate treatment. However, it is unexpected that the recovery of both the MD value and $P$ value lagged behind that of visual acuity. The visual field results are in general accordance with those presented by Gordon et al. [20] in birdshot choroiretinopathy. They also found that the visual field test is independent of other measures of visual dysfunction and that visual field recovery lagged behind that of visual acuity. These observations may suggest important features concerning the pathology of both diseases. More studies are needed to clarify this issue. An unsynchronized recovery of visual acuity, central visual field and color vision in patients with VKH syndrome suggests that sub-clinical abnormal macular function is still present in certain patients although the intraocular inflammation is completely controlled.

In summary, our studies showed that a severely decreased visual acuity was associated with abnormal color vision and visual field defects in patients with active VKH syndrome. Following appropriate immunosuppressive treatment, improvement of visual acuity and color vision was faster than that of the visual field. Both color vision and visual field parameters may provide additional quantifiable measurements of visual dysfunction in patients with VKH syndrome. However, it is not yet known whether visual field or color vision at baseline is a better predictor of final visual acuity. Further studies are needed to clarify this issue.

Acknowledgement This work was mostly carried out at Zhongshan Ophthalmic Center. We thank Prof. Dezheng Wu, Prof. Feng Wen, Prof. Shizhou Huang, Prof. Guangwei Luo, Prof. Shixian Long and Mr. Futian Jiang for their support in this study.

Declaration of interest None of the authors has a proprietary or financial interest in any product mentioned.

Open Access This article is distributed under the terms of the Creative Commons Attribution License which permits any use, distribution, and reproduction in any medium, provided the original author(s) and the source are credited.

\section{References}

1. Forster DJ, Cano MR, Green RL et al (1990) Echographic features of the Vogt-Koyanagi-Harada syndrome. Arch Ophthalmol 108:1421-1426

2. Moorthy RS, Inomata H, Rao NA (1995) Vogt-Koyanagi-Harada syndrome. Surv Ophthalmol 39:265-292

3. Murakami S, Inaba Y, Mochizuki M et al (1994) A nation-wide survey on the occurrence of Vogt-Koyanagi-Harada disease in Japan. Nippon Ganka Gakkai Zasshi 98:389-392

4. Read RW, Rechodouni A, Butani N et al (2001) Complications and prognostic factors in Vogt-Koyanagi-Harada disease. Am J Ophthalmol 131:599-606

5. Yang P, Ren Y, Li B et al (2007) Clinical characteristics of VogtKoyanagi-Harada syndrome in Chinese patients. Ophthalmology 114:606-614

6. Chee SP, Luu CD, Cheng CL et al (2005) Visual function in VogtKoyanagi-Harada patients. Graefes Arch Clin Exp Ophthalmol 243:785-790

7. Yang P, Fang W, Wang L et al (2008) Study of macular function by multifocal electroretinography in patients with Vogt-KoyanagiHarada syndrome. Am J Ophthalmol 146:767-771

8. Hidajat RR, Hidayat JR, McLay JL et al (2004) A fast system for reporting the Farnsworth-Munsell 100-hue colour vision test. Doc Ophthalmol 109:109-114 
9. Read RW, Holland GN, Rao NA et al (2001) Revised diagnostic criteria for Vogt-Koyanagi-Harada disease: report of an international committee on nomenclature. Am J Ophthalmol 131:647-652

10. Holladay JT (2004) Visual acuity measurements. J Cataract Refract Surg 30:287-290

11. Huang S, Wu L, Wu DZ (1993) The normal color vision evaluated with FM 100-hue test. Yan Ke Xue Bao 9(158-160):145

12. Rubsamen PE, Gass JD (1991) Vogt-Koyanagi-Harada syndrome. Clinical course, therapy, and long-term visual outcome. Arch Ophthalmol 109:682-687

13. Magacho L, Henderer JD, Lankaranian D et al (2006) Improvement in colour vision parameters following successful trabeculectomy. Acta Ophthalmol Scand 84:201-205

14. Botstein D (1986) The molecular biology of color vision. Science 232:142-143

15. Barton FB, Fong DS, Knatterud GL (2004) Classification of Farnsworth-Munsell 100-hue test results in the early treatment diabetic retinopathy study. Am J Ophthalmol 138:119-124

16. Vuori ML, Mantyjarvi M (2007) Tilted disc syndrome and colour vision. Acta Ophthalmol Scand 85:648-652

17. Menage MJ, Papakostopoulos D, Dean Hart JC et al (1993) The Farnsworth-Munsell 100 hue test in the first episode of demyelinating optic neuritis. Br J Ophthalmol 77:68-74
18. Pacheco-Cutillas M, Edgar DF, Sahraie A (1999) Acquired colour vision defects in glaucoma-their detection and clinical significance. Br J Ophthalmol 83:1396-1402

19. Holland GN, Shah KH, Monnet D et al (2006) Longitudinal cohort study of patients with birdshot chorioretinopathy II: color vision at baseline. Am J Ophthalmol 142:1013-1018

20. Gordon LK, Monnet D, Holland GN et al (2007) Longitudinal cohort study of patients with birdshot chorioretinopathy IV. Visual field results at baseline. Am J Ophthalmol 144:829-837

21. Chan CC, Palestine AG, Kuwabara T et al (1988) Immunopathologic study of Vogt-Koyanagi-Harada syndrome. Am J Ophthalmol 105:607-611

22. Herbort CP, Mantovani A, Bouchenaki N (2007) Indocyanine green angiography in Vogt-Koyanagi-Harada disease: angiographic signs and utility in patient follow-up. Int Ophthalmol 27:173-182

23. Arellanes-Garcia L, Hernandez-Barrios M, Fromow-Guerra $J$ et al (2007) Fluorescein fundus angiographic findings in Vogt-KoyanagiHarada syndrome. Int Ophthalmol 27:155-161

24. Yamaguchi Y, Otani T, Kishi S (2007) Tomographic features of serous retinal detachment with multilobular dye pooling in acute Vogt-Koyanagi-Harada disease. Am J Ophthalmol 144:260 265 